\title{
Intervention for Infants at Risk of Developing Autism: A Case Series
}

\author{
Jonathan Green - Ming Wai Wan · Jeanne Guiraud - Samina Holsgrove • \\ Janet McNally • Vicky Slonims • Mayada Elsabbagh • Tony Charman • \\ Andrew Pickles $\cdot$ Mark Johnson • The BASIS Team
}

Published online: 27 March 2013

(C) Springer Science+Business Media New York 2013

\begin{abstract}
Theory and evidence suggest the potential value of prodromal intervention for infants at risk of developing autism. We report an initial case series $(n=8)$ of a parent-mediated, video-aided and interaction-focused intervention with infant siblings of autistic probands, beginning at $8-10$ months of age. We outline the theory and evidence base behind this model and present data on feasibility, acceptability and measures ranging from parentinfant social interaction, to infant atypical behaviors, attention and cognition. The intervention proves to be both feasible and acceptable to families. Measurement across domains was successful and on larger samples promise to be an effective test of whether such an intervention in infancy will modify emergent atypical developmental trajectories in infants at risk for autism.
\end{abstract}

The BASIS team members are as follows: Simon Baron-Cohen, Patrick Bolton, Kim Davies, Janice Fernandes, Helena Ribeiro, and Leslie Tucker.

Electronic supplementary material The online version of this article (doi:10.1007/s10803-013-1797-8) contains supplementary material, which is available to authorized users.

\section{J. Green $(\bowtie)$}

Institute of Brain, Behaviour and Mental Health, University of Manchester, Room 4.308, Jean McFarlane Building, Oxford Rd, Manchester M13 9PL, UK

e-mail: jonathan.green@manchester.ac.uk

M. W. Wan · S. Holsgrove · J. McNally

Institute of Brain, Behaviour and Mental Health, University of Manchester, Jean McFarlane Building, Oxford Rd,

Manchester M13 9PL, UK

J. Guiraud · M. Elsabbagh · M. Johnson

Centre for Brain and Cognitive Development, Department of Psychological Science, Birkbeck, University of London,

The Henry Wellcome Building, London WC1E 7HX, UK
Keywords Autism - Intervention · Prodromal - Infancy · Parent-child interaction

\section{Introduction}

Recent theoretical models of the early emergence of autism spectrum disorder (ASD) have proposed that infant intrinsic risk susceptibilities in brain functioning or behaviour may be amplified by interaction in the early social environment into an increasingly atypical developmental trajectory (Dawson 2008; Elsabbagh and Johnson 2007; Elsabbagh and Johnson 2010). Part of the appeal of such models is their dynamic view of development and the corresponding possibilities they imply for early intervention (Dawson 2008; Wallace and Rogers 2010). 'Interactive specialization' theory in developmental neuroscience has detailed how postnatal social experience may influence the specialization and localization of the neural substrates of the developing 'social brain': typically developing infants and toddlers are biased to orient towards, attend to, and learn

\footnotetext{
V. Slonims

Guy's and St Thomas' NHS Foundation Trust/King's College

London, Great Maze Pond, London SE1 9RT, UK

\section{T. Charman}

Department of Psychology, Institute of Psychiatry,

King's College London, Box PO77, Henry Wellcome Building,

De Crespigny Park, Denmark Hill, London SE5 8AF, UK
}
A. Pickles
Department of Biostatistics, Institute of Psychiatry,
King's College London, London SE5 8AF, UK 
from, social stimuli and these biases contribute to the progressive post-natal emergence of a typical cortical social brain network (Johnson 2001, 2011). In ASDs it is assumed that an intrinsic failure or abnormality in one or more of the underlying mechanisms of such biasing from infancy disrupts the typical emergence of the social brain network. An initial atypical trajectory may then become further amplified by interactions within the social environment, leading to the emergence of an established pattern of symptoms in social and communicative functioning observable at diagnosis, which is rarely before the age of 2 years (Charman and Baird 2002; Chawarska et al. 2009). This would not suggest then that these interactional cycles were a primary cause of autism, but rather that they might maintain or amplify a preexisting vulnerability.

Support for such theory comes from research in developmental psychology and psychopathology, demonstrating the importance of the early social interactive environment on the general development of joint attention, reciprocity, and mutuality, and hence effects on later socialisation and communication (NICHD 2000; Murray et al. 1996). Atypical neurodevelopment (for instance in Down syndrome, cerebral palsy, learning disability) impacts on this social interaction; associated, for instance, with generally reduced parental sensitivity to infant behavioural signals and increased 'intrusiveness' in relation to the infant (Cardoso-Martins and Mervis 1985; Crawley and Spiker 1983), perhaps arising from adult difficulty in accurately interpreting infant behaviours (Sorce and Emde 1982; Dunst 1985; Slonims et al. 2006). A directive parental style is not incompatible with sensitivity (McCathren et al. 1995), but supportive, contingent and sensitive parental responses are central to the development of joint attention: later language development is accelerated when the adult follows child-initiated and child-focused topics for joint attention (McCathren et al. 1995; Harris et al. 1996). This may be particularly important in children whose developmental impairments lead to difficulties in accommodating demands to shift focus and to regulate several competing demands on attention (Legerstee et al. 2002; Yoder and Warren 2004; Walden et al. 1997).

These theoretical considerations imply that a truly prediagnostic preventative intervention strategy at a behavioural level may be feasible in autism and that a logical approach would be to target known developmental processes that may act to amplify atypical development (Dawson 2008; Wallace and Rogers 2010), especially if these are known to be potentially sensitive to intervention. In this context, our developmental studies of early parent-infant interaction in infants at familial high risk of autism compared to low risk controls without an older sibling with ASD (Wan et al. $2012 \mathrm{a}, \mathrm{b}$ ) have shown that high risk dyads exhibit a reduced level of parental sensitive responding, increased parental directiveness and reduced infant attentiveness, affect and liveliness at 8 months; and that reduced dyadic mutuality, infant attentiveness and infant positive affect at 12 months predicts ASD outcome at 3 years. These findings are consistent with retrospective study of parents' home videos suggesting that specific directive behaviours (longer stimulatory behaviour, and more use of touch to elicit attention) differentiated parents whose infants were later diagnosed with ASD $(n=15)$ from parents of typically developing infants and infants with intellectual disabilities (SaintGeorges et al. 2011). It has also been previously reported that 4-month-old at-risk siblings $(n=21)$ showed less affectively synchronous interactions when infants led play with their mothers compared to low-risk comparisons, suggesting that these mothers find it difficult to match infant-initiated affect (Yirmiya et al. 2006). However in other studies, atypical social orienting was not found at 6 months of age in at-risk infant who later went on to have a diagnosis of ASD ( $n=17$ ), although by 12 months they showed lower rates of joint attention and requesting (Rozga et al. 2011).

These findings lend empirical support to an intervention focusing on optimising parent-infant dyadic interaction. Furthermore, our own and others' work has shown that these aspects of parental behaviour and dyadic interaction are amenable to change with intervention both in the low and high autism risk context (see 'intervention' below). It is important to stress that any causal relationship (and its direction) between these interactive changes and child outcomes is yet to be established. With the appropriate design, testing an intervention of this kind could itself act as a strong test of this developmental model and putative causal relationships (Green and Dunn 2008; Aldred et al. 2011; Pickles et al. 2011). For instance, in a design using randomised group allocation along with longitudinal observational follow up, a focused intervention can act as a discrete developmental perturbation in one of the parallel groups and the longitudinal follow up can identify any impact of the perturbation on later development and test causal direction (Howe et al. 2002).

In addition to a focus on changing developmental trajectories at a behavioural level, the 'interactive specialisation' account of emergent autism (Elsabbagh and Johnson 2010) suggests that an environmental change in the infant's social environment might also be expected to show impact at the level of brain functional specialisation. This would be the most ambitious goal of a developmental intervention at this age, with potentially radical developmental consequences. Testing this hypothesis demands simultaneous measurement across related domains of infant behaviour, parent-infant interaction, infant cognitive and brain function.

Because of the relatively low baseline prevalence of ASD, a truly preventative strategy of this individualized kind needs to be 'indicated' rather than 'universal'; that is, targeting groups of infants or young children at particular 
risk of developing autism rather than the whole population. The latter would be quite impracticable for $1 \%$ of the population, whereas the rates of development of ASD in the high risk sibling group is found to be approximately $20 \%$ in substantial prospective cohort studies (Ozonoff et al. 2011), making feasible the targeting and testing of a high-risk 'indicated' preventative approach of this kind. The timing of such prodromal intervention needs to balance a maximizing of the theoretical potential of brain plasticity at the earliest point on the one hand with the gradual emergence of atypicality and current lack of identifiable predictive markers for autism at very early ages on the other. Convergent evidence from high-risk infant sibling studies indicates that the $8-14$ month period is notable for the earliest emergence of such identifiable social interaction and behavioural atypicalities, while more definite symptom emergence is often not seen until 18 months or later (Yirmiya and Charman 2010).

Together, these arguments give convergent logic for an evidence-based intervention focusing on parent-infant dyadic interaction in the 8-14 month period with infants at high risk of developing autism, using simultaneous measures across domains. A recent 3-case series (Steiner et al. 2013) has reported on an intervention at 12 months. This paper presents a case series report of such a prevention model in a high-risk infancy group beginning at $8-10$ months. Within this, we describe the empirical and theoretical foundations of the intervention itself, and rationale for the measures usedas well as findings from an initial case series study on feasibility and acceptability, including cross-domain developmental measures that range across infant behavior and development, parent-infant social interaction, and a visual attention task.

\section{Methods}

\section{Participants}

All participants were recruited from families within the British Autism Study of Infant Siblings (BASIS; www.basisnetwork.org), involving families with infants at risk of developing autism by virtue of having an older sibling with an autism spectrum disorder and families with infants at low risk by virtue of the absence of reported ASD in other members of the family. Families self-refer after advertisement or are identified within clinical services, and provide generic information to the database as well as that specifically for this study. Full details of procedures and information have been described separately (Elsabbagh et al. 2012; Bedford et al. 2012).

The Study group $(n=8)$ for the intervention case series were recruited from the high-risk infant group in BASIS when infants were 8-10 months old. We did not select by presence or absence of any observed atypicality in these infants at this time; the meaning and status of atypical behaviours at this age remains uncertain and we considered it most scientifically and ethically appropriate to use an unselected sample. Currently published candidate precursors to diagnosis in toddlerhood have mostly been analyzed at the group level and their sensitivity and specificity at an individual level have yet to be determined. One family in the series, who had three older children with autism, became un-contactable after the first intervention session for reasons we were unable to establish. The remaining seven families completed the intervention and all assessments, and their data are reported here.

Data on this intervention case series are compared with data from two comparison groups

1. A high-risknon-intervention group - unselected infants from the BASIS cohort within the same age range with comparable data on measures used. They were included as a high-risk reference to index how intervention group trajectory might compare with trajectories from similar high-risk infants on each task.

2. A low-risk non-intervention group-infants of comparable age range but without an older sibling with autism. This control group was intended to serve as a reference group in relation to normative development on each task; to assess whether intervention moved atypical trajectory indices in our risk group towards a normative range.

Infants at-risk $(n=44 ; 7$ intervention cases and a total of 37 high risk non-intervention comparison cases) were all defined by having at least one older sibling (hereafter, proband) with a community clinical diagnosis of ASD (in 1 case, a half-sibling; 36 were male, 8 female). This proband clinical diagnosis was confirmed by expert clinical consensus diagnosis (PB, TC) using triangulated information within the Development and Wellbeing Assessment (DAWBA, Goodman et al. 2000) and the parent-report Social Communication Questionnaire (SCQ, Rutter et al. 2003). Most probands met ASD criteria on both the DAWBA and SCQ. In a minority of probands with community clinical diagnosis but data only available for either the DAWBA or the SCQ $(n=13)$, consensus diagnosis was made using ASD criteria met on one measure $(n=8)$ or reported clinical diagnosis alone $(n=5)$. Parent-reported family medical histories were examined for significant medical conditions in the proband or extended families members, with no exclusions made on this basis. At the time of enrolment, none of the infants had been diagnosed with any medical or developmental condition. Infants in the low-risk group (total $\mathrm{N}=33$ ) were recruited from a volunteer database at the Birkbeck Centre for Brain and 
Cognitive Development. Inclusion criteria included fullterm birth, normal birth weight, and lack of any ASD within first-degree family members (as confirmed through parent interview regarding family medical history). All low-risk infants had at least one older-sibling (in 3 cases, only halfsibling/s). 18 of the older siblings were male, 15 were female. Screening for possible ASD in these older siblings was undertaken using the SCQ $(\mathrm{N}=27$ returned), with no child scoring above instrument cut-off for $\operatorname{ASD}(\geq 15)$.

\section{Procedures}

Informed consent was obtained for this intervention study. Baseline assessment took place between 8 and 10 months at the Centre for Brain and Cognitive Development, Birkbeck, London. Within 1 month of this, a 5 month (12 session) home-based intervention programme began. Endpoint assessments took place at 14-15 months. All sessions were video and audiotaped. The intervention study received specific multisite ethical approval (09/HO718/14) as well as local site approvals and was conducted in accordance with the Declaration of Helsinki (1964).

\section{Intervention}

The 'Intervention in BASIS' (iBASIS) programme is based on three levels of theory and evidence. Firstly, in line with the developmental context and theoretical rationale for this targeted prevention strategy, we grounded the intervention in a generic programme applicable to parents and infants with or without neurodevelopmental atypicality. Meta-analysis of 81 studies of infancy interventions ( $\mathrm{n}=7,636$; BakermansKranenburg et al. 2003) suggests personalised video aided intervention with parents to be most effective in improving early parental responsiveness to infants (overall, $d=0.45$ ) and best delivered in the latter part of the first year $(\mathrm{d}=0.44)$. The manualised Video Interaction for Promoting Positive Parenting (VIPP; Juffer et al. 2008) is one of the best studied of these methods across a range of risk conditions (although not previously autism). It has a strong evidence base for significant alteration of relevant aspects of parental interactive behaviour along with moderate effect on child outcomes (Juffer et al. 2008; Bakermans-Kranenburg et al. 2003). Adaptations to VIPP have recently been made by the originators for use in an ongoing study of diagnosed preschool children with autism (van IJzendoorn et al. personal communication, June 2011); relevant adaptations are also reflected in our infancy model. Into this base we integrated autism-specific intervention procedures shown in our Preschool Autism Communication Trial (PACT; Green et al. 2010, Pickles et al. 2011; Aldred et al. 2011) to have a rapid and substantial impact on parental communicative synchrony behaviour with the child with autism (Effect Size
(ES) 1.44 at 6 months; 1.22 at 13 months); with this change in parental synchrony mediating downstream improvements in child dyadic communication (ES 0.5 at 6 months, 0.44 at 13 months) and modest gains in ADOS symptom score at 13 months (ES 0.24). This detailed effectiveness and causal mediation evidence is consistent with other early autism intervention work (Kasari et al. 2010) and with the developmental literature in that high early parental synchrony is associated with enhanced later language and social development in both neuro-typical and autistic children (Siller and Sigman 2002, 2008). A final level of evidence base for iBASIS comes from our own empirical studies showing differences in parent-infant social interaction in the situation of infant siblings at high risk for autism compared to infant controls at low risk for autism both at 7-9 months and 12-15 months, with the latter associated with later ASD diagnosis (Wan et al. 2012a, b). These findings support the primary theory of early autism development described in the introduction and provide the rationale for an intervention modifying these effects. The iBASIS manual was further adapted to include specific procedures to deal with any observed atypicality during the course of intervention. The resulting intervention is thus an amplified and extended version of the basic VIPP programme, with 12 home-based sessions over 5 months. Sessions are initially weekly and then reduced in frequency. The parent is encouraged to undertake $30 \mathrm{~min}$ of structured practice each day between sessions. The intervention is parent-mediated and videoaided with no direct contact between the infant and therapist (see online resource 1 for details of the intervention protocol).

\section{Measures}

\section{Intervention Acceptability (Endpoint)}

Acceptability of the intervention was assessed through an eleven-item self-rated parent questionnaire consisting of stem questions with five responses (strongly agree, agree, neutral, disagree, strongly disagree) and opportunity for additional text comments. Items covered included whether expectations of intervention were met; whether session number, length, frequency and content were appropriate; session benefits, or negative consequences; impact on wider family; potential improvements to intervention. Additional qualitative information was obtained through interviews between parents and therapist following completion of therapy, which were videotaped, transcribed and subjected to thematic analysis.

\section{Caregiver-Infant Interaction (Baseline and Endpoint)}

We hypothesised that the intervention would influence the quality of interaction between parents and their child; 
specifically that the quality of parental synchronous response to infant communication would be enhanced (in line with the findings in Green et al. 2010). To test this, parent-infant play interaction episodes were analysed from video recordings taken at the assessment visit pre- and post-intervention ('lab interaction') and during the first and last home-based therapy sessions ('home interaction'). The lab measure was taken since the physical set-up could be standardised and was used also across HR and LR comparison groups. The home based video recordings were recorded as an intrinsic part of the therapy procedure (see above) and were analysed in this study as a test of the representativeness of the lab video recordings vis-a-vis naturalistic play in the home in the context of therapy. Lab play sessions involved the parent and infant seating on a floor mat in a room, following a period of familiarisation with the environment. The parent was instructed to engage in play with the infant, as she would do at home, using a set of toys provided if they so wished. Home-based sessions were set up in a similar way. The first 6 min of the video clips were independently rated, blind to participant information and time point. The control groups were rated blind to participant information and group/risk status. The Manchester Assessment of Caregiver Infant Interaction (MACI; Wan et al. 2012a, b) evaluates global aspects of unstructured play interaction important for socioemotional development in infants 6-15 months. It consists of seven scales adapted for this specific age-range from two previous measures; the Global Rating Scales (Murray et al. 1996), developed for younger infants up to 6 months and the Coding of Attachment-Related Parenting in Autism (CARP-A; Matias 2006; Blazey 2007), developed for toddlers and pre-schoolers. Interaction is rated along a 1 (lowest) to 7 (highest) scale on 2 parent scales (sensitive responsiveness, non-directiveness), 3 infant scales (attentiveness to parent, positive affect, and liveliness), and 2 dyadic scales (mutuality and intensity of engagement). Details of the operationalization of these interaction variables are detailed in online resource 2 . Inter-rater agreement was high in both high- and low-risk comparison groups (Wan et al. 2012a, b); and was further tested in the intervention group by blinded double coding of $56 \%$ of clips, using single measures intraclass correlation (two-way mixed effects model with an absolute agreement definition), with moderate to high agreement (all $p<0.001$ ) on all coding items in both home and lab contexts (range of agreement in home observations: $r=0.70-0.90$; lab observations: $r=0.69-0.93$ ).

\section{Infant Developmental and Adaptive Level (Baseline and Endpoint)}

The Mullen Scales of Early Learning (Mullen, 1995) is a standardized, norm-referenced, developmental evaluation valid to 68 months-including subscales of: receptive and expressive language, visual reception, gross and fine motor skills, and an early learning composite quotient. In this report we focus in the expressive and receptive language raw scores, which we could expect to reflect any change over this period.

\section{Infant Behaviour}

The Autism Observation Scale for Infants (AOSI; Bryson et al. 2008) was specifically developed to identify early putative behavioural features of autism between 6 and 18 months. It is structured and observer rated during researcher-infant interaction. Emerging evidence suggests that atypicality on AOSI is identified prior to 12 months in high-risk groups and becomes increasingly predictive thereafter; with 12 month AOSI scores predictive of later diagnoses of core autism with learning disability, although not of the wider ASD spectrum (Zwaigenbaum et al. 2011; higher AOSI scores equate to more atypicality). By including the AOSI in the protocol we hope to ascertain whether emerging symptoms of autism can be influenced by the intervention.

\section{Infant Visual Attention: The Gap-Overlap Task}

Laboratory measures reflecting the development of attention skills may be useful in the context of intervention studies, and it remains unknown whether or not performance in these tasks would be influenced by parentmediated intervention. There is growing consensus in infant siblings research that early emerging behavioral signs of autism and the broader phenotype include a range of social and non-social skills (reviewed in Elsabbagh and Johnson 2010; see recent examples in Flanagan et al. 2012; Chawarska et al. 2012). One such candidate is the Gapoverlap task, which involves measuring saccadic reaction time to peripheral visual targets with, or without, a competing central stimulus (Johnson et al. 1991). In this task a central stimulus is presented followed by a peripheral target stimulus either during continuing presentation of the central stimulus (overlap trials), or after the offset of the central fixation stimulus (baseline trials). The additional reaction time taken to orient to the peripheral target in the overlap trials is thought to be due to the need to disengage attention from the fixation stimulus (disengagement cost). When a temporal gap precedes the peripheral target in gap trials, infants are cued and would orient faster after its onset (facilitation). Zweigenbaum et al. (2005) presented preliminary evidence that difficulty in disengaging from a centrally presented visual stimulus could be an early marker for later emerging ASD symptoms. Our studies using visual attention tasks with infants at-risk, suggest that early 
disengagement skills in the first year of life map onto autism diagnosis (Elsabbagh et al. in press) as well as social-communication impairment as measured by the ADOS (Elsabbagh et al. 2011) in toddlerhood. Therefore, including the gap-overlap task as measure of response to intervention is consistent with previous findings suggesting that attention and social skills are inter-dependent over development in autism.

With only minor differences, the same task and analytic procedure was used in the current case series as those previously reported (Elsabbagh et al. in press). Infants were presented with the stimuli on a monitor, while seated on their parent's lap. Looking behavior was monitored and recorded through video from an adjacent room. All trials in this task began with a centrally presented animation. The peripheral target was presented randomly either to the right or the left of the central fixation stimulus. Peripheral targets were always the same (a dynamic green balloon) and remained displayed until the infant looked at them or until 3 s elapsed. Once the infant looked to the target or if the maximum duration was reached, an attractive animation of an animal with sound replaced the peripheral target. In the Baseline condition, the central fixation stimulus was extinguished and the peripheral target appeared simultaneously; in the Gap condition the fixation stimulus disappeared $200 \mathrm{~ms}$ before the peripheral target; in the Overlap condition the animated peripheral target appeared while the central fixation stimulus remained displayed (but not animated) so that the two stimuli overlapped. The three conditions were presented randomly across two blocks of 35 trials. The two blocks were identical except for the central fixation stimulus to maintain the infant's interest in the task. Trial presentation continued until the infant became fussy or until a maximum of 70 trials was reached. Across the three conditions, infants completed on average 42 valid trials during the first visit $(\mathrm{LR}=43.1, \mathrm{HR}=41.8$, Intervention $=40.8$ ) and 38 valid trials during the second visit $(\mathrm{LR}=38.1, \mathrm{HR}=37.8$, Intervention $=36.6)$.

\section{Analysis}

In this case series report we first present data related to feasibility and acceptability and descriptive data on prepost measures at case-level. We then present change scores on each measure over the intervention period using the available data from the two comparison groups ( $\mathrm{N}$ for each measure detailed in tables and figures). We calculated change scores on each measure within HR and LR groups and two standardized change scores on each measure for intervention children by subtracting a mean and dividing by a SD. In the first instance this was the mean and SD of the LR change scores, and in the second the mean and SD of the HR change scores. Thus, a score above 0 implied greater change than observed for the mean of data for that reference group. We used a cut-off of $\pm 1.5 \mathrm{SD}$ as an indicator of change potentially different from the normal distribution of change.

\section{Results}

\section{Participant Characteristics}

Descriptive data on completed intervention and the two comparison groups is shown in Table 1. All intervention families were 2 parent households with the mother not working at the time of the intervention, in one family both parents were not working. Data from the two control groups show no group differences in infant age, but a higher proportion of infants in the intervention group had more than one older sibling. The intervention in all cases involved the mother.

\section{Intervention Feasibility}

All therapeutic sessions took place at home, were conducted by one of the two study therapists, and lasted about $1.5 \mathrm{~h}$. Five of seven families attended all twelve sessions, one family ceased after 10 sessions (by mutual agreement since they felt they had achieved their goals), and a further family completed 11 sessions. In five of seven families the older autistic proband was in nursery or school at the time of the arranged sessions and this eased the delivery of the intervention. In one family with a pre-school affected sibling an assistant accompanied the therapist on home visits. In another family with two siblings with autism, one of whom was not always at school, the father assisted during therapy visits. With these arrangements the home-based protocol proved quite feasible to deliver. Families were asked to undertake $30 \mathrm{~min}$ focused practice per day; families sometimes said this was difficult to achieve, but none of the seven families in the series said this was impossible.

\section{Acceptability}

Information on acceptability was obtained from self-completed questionnaire report. All seven parents strongly agreed the intervention was enjoyable and that it had led to a greater understanding of their infant's behaviours; two of seven that the intervention had exceeded their expectation of benefit. All parents either strongly agreed $(n=6)$ or agreed $(\mathrm{n}=1)$ that the intervention was helpful; and all either strongly agreed $(n=3)$ or agreed $(n=4)$ that sessions were of appropriate number and duration. All parents either strongly agreed $(n=5)$ or agreed $(n=2)$ that they had modified aspects of their parenting as a result of the 
Table 1 Sample characteristics

\begin{tabular}{|c|c|c|c|c|}
\hline & \multicolumn{2}{|c|}{ High risk intervention $\left(\mathrm{N}=7^{\mathrm{a}}\right)$} & \multirow{2}{*}{$\begin{array}{l}\text { High risk comparison } \\
(\mathrm{N}=37)\end{array}$} & \multirow{2}{*}{$\begin{array}{l}\text { Low risk comparison } \\
(\mathrm{N}=33)\end{array}$} \\
\hline & Lab & Home & & \\
\hline \multicolumn{5}{|l|}{ Mean infant age months (SD) } \\
\hline Visit 1 & $8.4(0.79)$ & $8.7(0.95)$ & $8.7(0.82)$ & $8.4(0.94)$ \\
\hline Visit 2 & $14.9(1.22)$ & $13.9(1.3)$ & $15.1(1.15)$ & $14.8(0.90)$ \\
\hline Boys $(\%)$ & $4(57.1 \%)$ & & $20(54.1 \%)$ & $16(48.5 \%)$ \\
\hline Number with $>1$ older sibling & $4(57.1 \%)$ & & $23(62 \%)$ & $17(51.5 \%)$ \\
\hline
\end{tabular}

${ }^{a}$ One family only completed one session and therefore not included here (see text)

programme, that the home environment for intervention was suitable and that they now spent more quality-time with their child. No parents identified negative effects from participating and four parents indicated they had been able to share some of their learning and/or some of the video from sessions with partners or the infant's older siblings.

Supplementary qualitative information was obtained at post-therapy interview with therapists. All parent interviews reported increased awareness of interaction with their infant and of their infant's communication with them; "It makes you look really closely at what your interaction with your child is and there is much more in it than you really thought". "It's amazing watching back how much she understands and how much she's taking in and communicating with you and you just don't notice it". Three parents commented that they had found reviewing the videos the most helpful thing in terms of session content. One mother reported that she found the session involving nursery rhymes and songs less comfortable but said that: "Because I felt uncomfortable doing it-it made me realise, I probably don't do this enough". Additional benefits of taking part included increased recognition of the infant's emotionality and intentionality: "It made me aware he does have feelings and he gets them across. Before I ignored it and thought it's just baby grunting"; "I was like-maybe he hasn't yet developed emotions... But through the sessions I found out that actually, no it was just the way that you do things and now he's full of energy." Three parents felt awkward about being videoed initially but indicated that this diminished over time. One parent indicated that it may have been helpful to have had more explicit direction around interacting with her baby, another that it might be good for the therapist to bring an activity for her other child, so that she did not feel excluded, and a third that it would be good to be given additional ideas for booster activities.

\section{Parent-Infant Interaction}

Figures 1 and 2 summarise the results on parent-infant interaction over the intervention period, comparing HR and LR non-intervention controls. The figures show that absolute ratings were consistently higher in the home setting than the lab context although the trajectory slopes were similar. More detailed analysis of these interaction results compared to controls using z scores are summarized in Table 2. On parent sensitive responding and non-directiveness, three of seven cases showed positive change scores in parent sensitive responding at near or above threshold of $1.5 \mathrm{SD}$ in relation to both control groups; and two cases showed the same on nondirectiveness. Results for infant interaction measures are detailed in online resource 3 .

The baseline level of functioning in intervention families is also a relevant context for these findings-in six of the seven intervention families, mothers were rated at a low
Fig. 1 Lab parent sensitivity pre-post intervention by mean and case

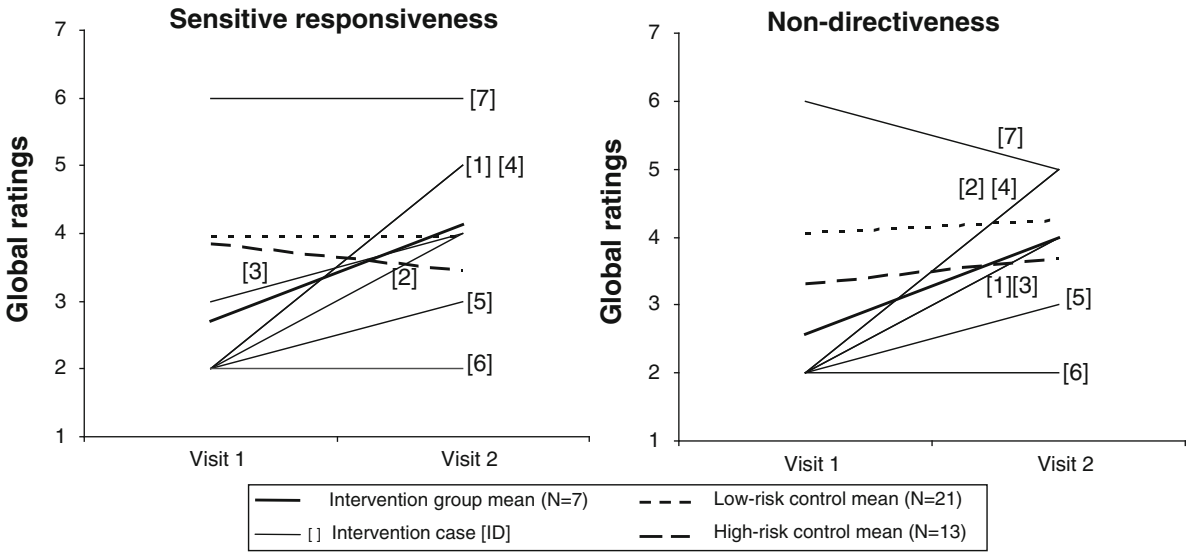


Fig. 2 Home parent sensitivity pre-post intervention by mean and case

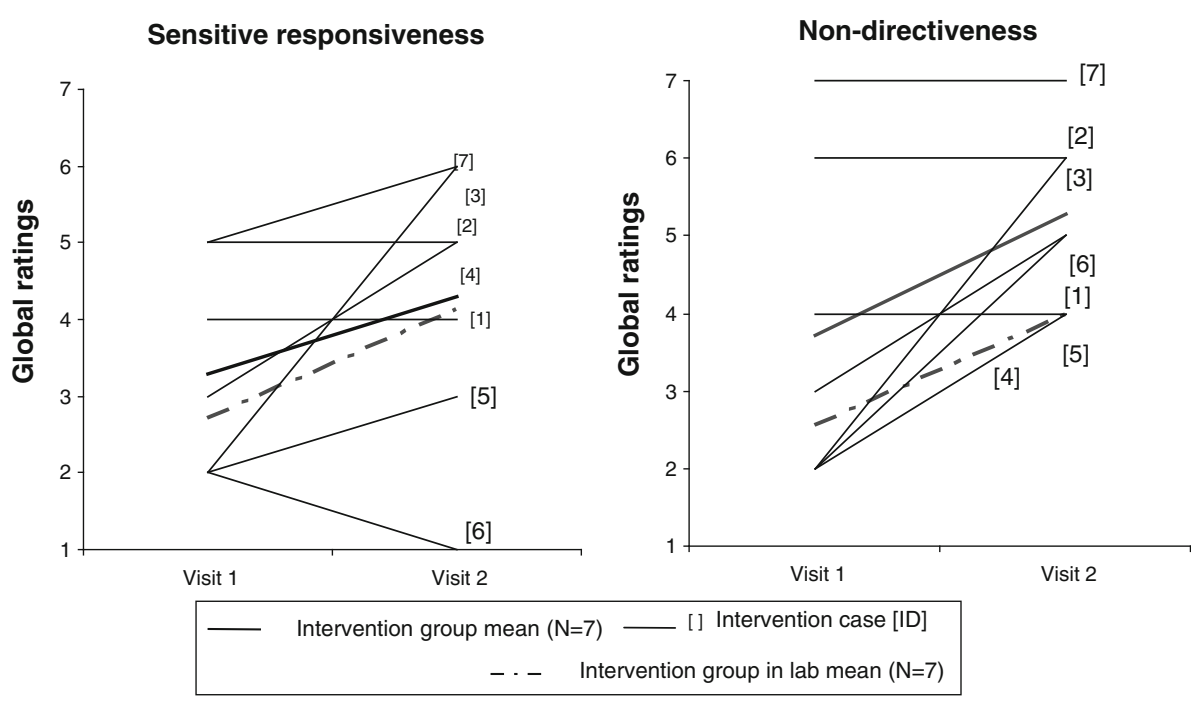

Table 2 Parent-infant interaction pre-post intervention: raw change scores and change expressed as Z scores compared with high and low risk group means

\begin{tabular}{|c|c|c|c|c|c|c|c|c|}
\hline \multirow[t]{4}{*}{ Case } & \multicolumn{8}{|l|}{ Parent } \\
\hline & \multicolumn{4}{|c|}{ Sensitive responsiveness } & \multicolumn{4}{|c|}{ Non-directiveness } \\
\hline & \multirow[t]{2}{*}{ Visit 1} & \multirow[t]{2}{*}{ Visit 2} & \multicolumn{2}{|c|}{ Change expressed as $\mathrm{Z}$ scores compared to } & \multirow[t]{2}{*}{ Visit 1} & \multirow[t]{2}{*}{ Visit 2} & \multicolumn{2}{|c|}{ Change expressed as $\mathrm{Z}$ scores compared to } \\
\hline & & & $\mathrm{HR}(\mathrm{n}=13)$ & $\operatorname{LR}(\mathrm{n}=21)$ & & & $\operatorname{HR}(\mathrm{n}=13)$ & $\operatorname{LR}(\mathrm{n}=21)$ \\
\hline 1 & 2 & 5 & 2.55 & 2.24 & 2 & 4 & 0.91 & 1.18 \\
\hline 2 & 2 & 4 & 1.80 & 1.49 & 2 & 5 & 1.49 & 1.83 \\
\hline 3 & 3 & 4 & 1.04 & 0.75 & 2 & 4 & 0.92 & 1.18 \\
\hline 4 & 2 & 5 & 2.55 & 2.24 & 2 & 5 & 1.49 & 1.83 \\
\hline 5 & 2 & 3 & 1.04 & 0.75 & 2 & 3 & 0.35 & 0.53 \\
\hline 6 & 2 & 2 & 0.29 & 0 & 2 & 2 & -0.22 & -0.12 \\
\hline 7 & 6 & 6 & 0.29 & 0 & 6 & 5 & -0.79 & -0.77 \\
\hline
\end{tabular}

Bold values indicate change $\mathrm{Z}$ score $\geq 1.5 \mathrm{SD}$

level ('2') in 'non-directiveness' at baseline; implying that the infant's autonomy and contribution to play would be clearly restricted as a result of the parent's low acceptance of the infant's behaviour and affect (this differs from the notion of structured support and active engagement of the infant). Five mothers were rated at a similarly low level ('2') for sensitive responsiveness, implying that sensitive responsiveness is seen only occasionally (i.e. there are either 2-3 moderate/partially responsive examples or 1 strong example in a 6 min interaction). All but one of these mothers showed an improvement on these parameters during the intervention period.

\section{Infant Behaviour-AOSI}

Of the intervention infants, Cases 5 and 7 had changing directions for their AOSI Total Score opposite to the ones observed in controls with changes falling outside 1.5SD of the average change in the HR group. However, both cases' values remained typical over time with scores falling within 1.5SD of the LR average range at both visits. Case 7's Total Marker Count also changed in the opposite direction to the ones observed in controls, with changes falling outside 2SD of both control group changes, and post-intervention value falling outside $2 \mathrm{SD}$ of the LR value (Table 3).

\section{Mullen}

Standardized score comparisons for the Mullen are shown in Table 4. In both control groups, Mullen average scores stay broadly stable. Case 6's change over time for the Early Learning Composite measure was outside 1.5SD of HR average change (this infant performed extraordinarily well following intervention with score falling outside $2 \mathrm{SD}$ of both control group ranges). Case 7's change on the Expressive Language measure fell outside 1.5SD of average change of both control groups. None of the intervention cases had changes on their Receptive Language measure 
Table 3 Raw pre- and post-intervention AOSI scores and changes expressed as Z scores compared with high and low risk group pre-post intervention mean changes

\begin{tabular}{|c|c|c|c|c|c|c|c|c|}
\hline \multirow[t]{3}{*}{ Case } & \multicolumn{4}{|c|}{ Total score } & \multicolumn{4}{|c|}{ Total marker count } \\
\hline & \multicolumn{2}{|c|}{ Raw scores } & \multicolumn{2}{|c|}{ Change expressed as $\mathrm{Z}$ scores compared to } & \multicolumn{2}{|c|}{ Raw scores } & \multicolumn{2}{|c|}{ Change expressed as $\mathrm{Z}$ scores compared to } \\
\hline & Visit 1 & Visit 2 & $\mathrm{HR}(\mathrm{n}=30)$ & $\operatorname{LR}(\mathrm{n}=27)$ & Visit 1 & Visit 2 & $\mathrm{HR}(\mathrm{n}=30)$ & $\operatorname{LR}(\mathrm{n}=27)$ \\
\hline 1 & 10 & 5 & -0.31 & -0.56 & 5 & 3 & -0.08 & -0.67 \\
\hline 2 & 2 & 3 & 0.71 & 0.30 & 1 & 2 & 0.98 & 1.02 \\
\hline 3 & 9 & 5 & -0.14 & -0.41 & 5 & 3 & -0.08 & -0.67 \\
\hline 4 & 4 & 6 & 0.88 & 0.44 & 3 & 4 & 0.98 & 1.02 \\
\hline 5 & 9 & 15 & 1.57 & 1.02 & 7 & 7 & 0.63 & 0.46 \\
\hline 6 & 10 & 7 & 0.03 & -0.27 & 7 & 4 & -0.44 & -1.23 \\
\hline 7 & 1 & 10 & 2.08 & 1.45 & 1 & 6 & 2.40 & 3.27 \\
\hline
\end{tabular}

Bold values indicate change $\mathrm{Z}$ score $\geq 1.5 \mathrm{SD}$

Table 4 Raw pre- and post-intervention Mullen scores and change expressed as Z scores compared with high and low risk group pre-post intervention mean changes

\begin{tabular}{|c|c|c|c|c|c|c|c|c|}
\hline \multirow[t]{3}{*}{ Case } & \multicolumn{4}{|c|}{ Expressive language } & \multicolumn{4}{|c|}{ Receptive language } \\
\hline & \multicolumn{2}{|c|}{ Raw scores } & \multicolumn{2}{|c|}{ Change expressed as $\mathrm{Z}$ scores compared to } & \multicolumn{2}{|c|}{ Raw scores } & \multicolumn{2}{|c|}{ Change expressed as $\mathrm{Z}$ scores compared to } \\
\hline & Visit 1 & Visit 2 & $\mathrm{HR}(\mathrm{n}=36)$ & $\mathrm{LR}(\mathrm{n}=31)$ & Visit 1 & Visit 2 & $\mathrm{HR}(\mathrm{n}=36)$ & $\mathrm{LR}(\mathrm{n}=31)$ \\
\hline 1 & 12 & 16 & -0.52 & -0.67 & 11 & 15 & -0.34 & -0.39 \\
\hline 2 & 10 & 18 & 0.94 & 1.02 & 12 & 20 & 0.81 & 0.57 \\
\hline 3 & 9 & 15 & 0.21 & 0.18 & 9 & 14 & -0.06 & -0.15 \\
\hline 4 & 9 & 14 & -0.15 & -0.25 & 10 & 15 & -0.06 & -0.15 \\
\hline 5 & 10 & 13 & -0.88 & -1.09 & 12 & 13 & -1.21 & -1.11 \\
\hline 6 & 10 & 15 & -0.15 & -0.25 & 11 & 15 & -0.34 & -0.39 \\
\hline 7 & 5 & 15 & 1.67 & 1.87 & 11 & 15 & -0.34 & -0.39 \\
\hline
\end{tabular}

Bold values indicate change $\mathrm{Z}$ score $\geq 1.5 \mathrm{SD}$

Fig. 3 AOSI pre-post intervention by mean and case

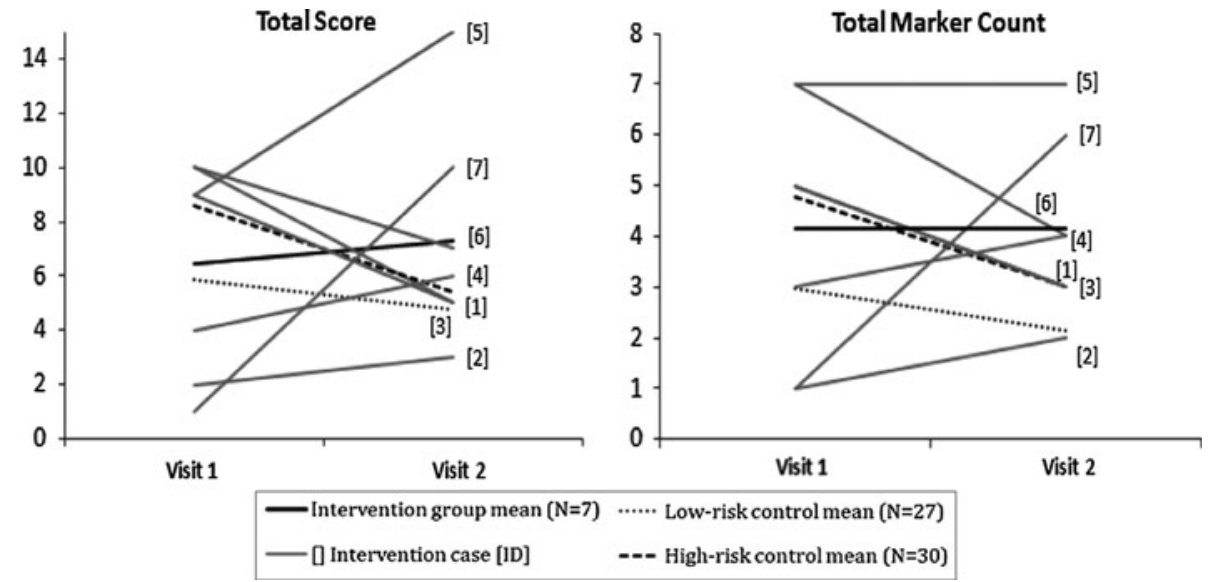

outside $1.5 \mathrm{SD}$ of the average changes of the control groups (Figs. 3, 4, 5).

\section{Gap-Overlap Task}

Based on their performance in the task, scores were calculated for each infant on disengagement and facilitation using the same procedure described in earlier studies (Johnson et al. 1991; Elsabbagh et al. 2009). Disengagement reflects the ability to disengage from a central stimulus to orient to a peripheral one. Results shown in Table 5 suggest that both low- and high-risk control groups showed slight decreases in disengagement latency between the two lab visits, supportive of previous evidence that the ability 
Fig. 4 Mullen pre-post intervention by mean and case
Fig. 5 Gap pre-post intervention by mean and case
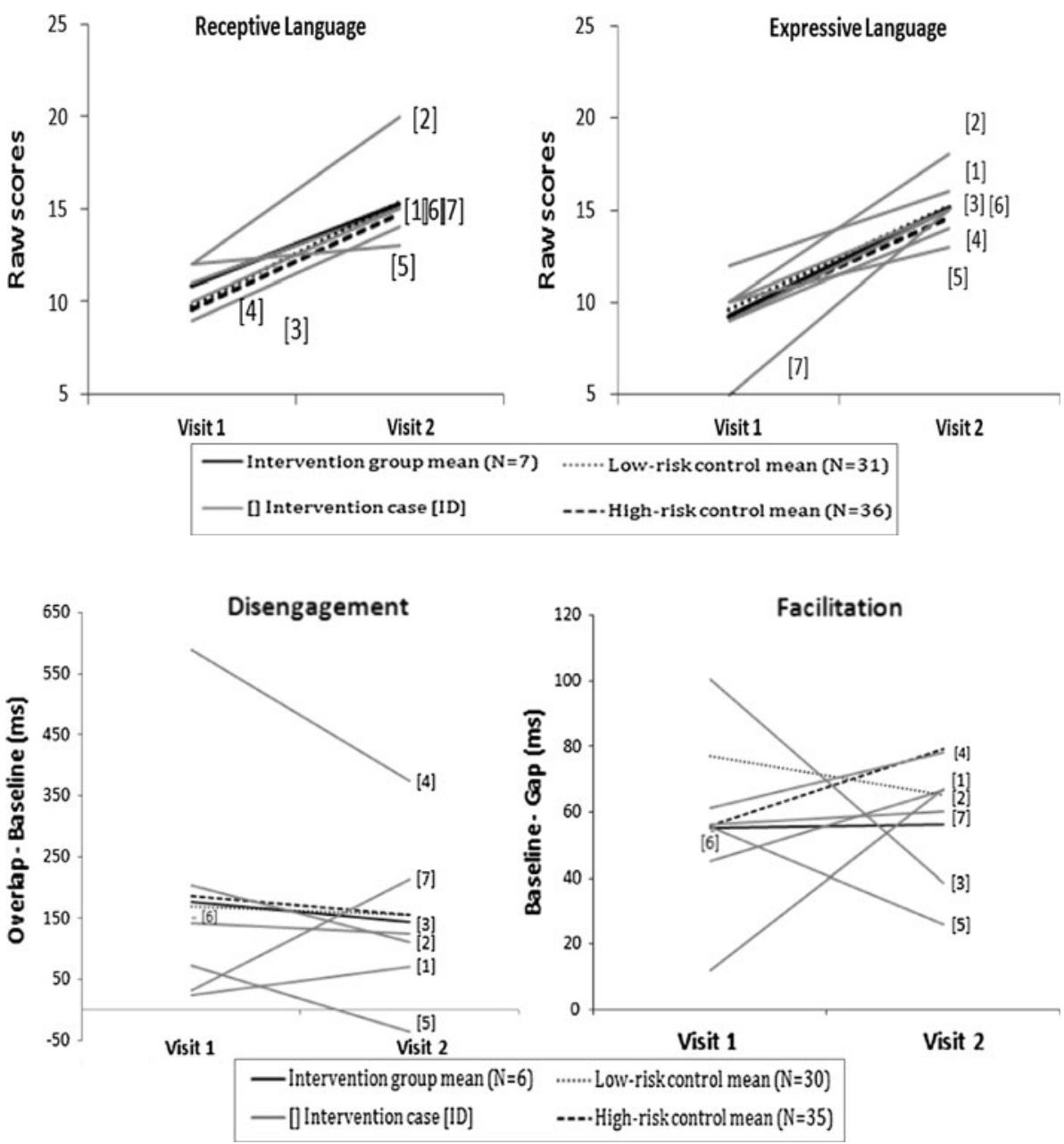

to disengage attention improves with age (Johnson et al. 1991). Data from one intervention infant (Case 6) was not available for visit 2 .

While no atypical values were observed for the case studies in the facilitation measure, for the disengagement measure two intervention infants had pre-post intervention changes outside 1.5 SD of the average change in the LR control group (Cases 4 and 7). For this measure only one intervention infant had an atypically large disengagement effect before the intervention commenced (Case 4). Although this infant disengagement latency decreased substantially following training, it still remained as an outlier compared to the control group data.

\section{Parent-Child Interaction in Relation to Other Measures}

There was no clear overall relationship in these cases between change in parent-infant interaction and assessed infant behavior. Case 7, for example, shows marked increase in AOSI scores (that is, increasing atypicality) but stable positive interactions. Of the other cases that show AOSI increases, Case 2 showed a substantial improvement in interaction (in mother and infant); Case 4 showed a large improvement in maternal scales (but slight decrease in infant attentiveness and mutuality); Case 5 showed small improvements in the maternal scales (but like Case 4, a slight decrease in infant attentiveness and mutuality).

\section{Discussion}

This case series represents to our knowledge the first description of a theoretically based intervention for infants at high risk of autism beginning within the first year of life. It uses a targeted prevention strategy that does not select for atypicality markers and measures across domains of infant behaviour and development, parent-infant interaction, family context, and cognition. An aim of this proof of concept study was to demonstrate the feasibility and acceptability of delivering the intervention at this age; and of taking simultaneous measures at these multiple levels. In this regard, the evidence from the number of sessions attended as well as independent parental questionnaire suggests that the intervention approach (12 sessions over 
Table 5 Raw pre- and post-intervention GAP scores and change expressed as Z scores compared with high and low risk group pre-post intervention mean changes

\begin{tabular}{|c|c|c|c|c|c|c|c|c|}
\hline \multirow[t]{3}{*}{ Case } & \multicolumn{4}{|c|}{ Disengagement (ms) } & \multicolumn{4}{|c|}{ Facilitation (ms) } \\
\hline & \multicolumn{2}{|c|}{ Raw scores } & \multicolumn{2}{|c|}{ Change expressed as $\mathrm{Z}$ scores compared to } & \multicolumn{2}{|c|}{ Raw scores } & \multicolumn{2}{|c|}{ Change expressed as $\mathrm{Z}$ scores compared to } \\
\hline & Visit 1 & Visit 2 & $\mathrm{HR}(\mathrm{n}=35)$ & $\mathrm{LR}(\mathrm{n}=30)$ & Visit 1 & Visit 2 & $\operatorname{HR}(\mathrm{n}=35)$ & $\mathrm{LR}(\mathrm{n}=30)$ \\
\hline 1 & 24.88 & 71.20 & 0.42 & 0.61 & 45.14 & 66.67 & 0.28 & 0.47 \\
\hline 2 & 204.79 & 111.24 & -0.35 & -0.80 & 11.98 & 67.03 & -0.19 & 0.19 \\
\hline 3 & 142.90 & 125.15 & 0.07 & -0.04 & 100.39 & 38.46 & 1.07 & 0.94 \\
\hline 4 & 589.67 & 373.39 & -1.03 & -2.03 & 61.15 & 78.14 & 0.51 & 0.60 \\
\hline 5 & 72.36 & -35.24 & -0.43 & -0.94 & 55.45 & 25.92 & 0.43 & 0.56 \\
\hline 7 & 31.47 & 213.85 & 1.17 & 1.98 & 56.41 & 60.14 & 0.44 & 0.56 \\
\hline
\end{tabular}

Bold values indicate change $\mathrm{Z}$ score $\geq 1.5 \mathrm{SD}$

5 months) is feasible to deliver in a home based context and acceptable to parents despite the fact they are in families already managing at least one child with autism and with potential concerns about a next born sibling. More nuanced concerns were addressed in detailed interviews with parents; whether, for instance, that intervening in this way might raise anxiety about the infant or introduce unacceptable burden on already busy families. We acknowledge the limitation that the therapists themselves conducted these interviews, raising the possibility that responses might be subject to bias towards acceptability. With this caveat, the interviews supported the positive evaluation from the questionnaires. All parents who completed the intervention said that they enjoyed it and that it provided them with an enriched sense of their infant's individuality and increased enjoyment in interaction. The parent responses are in keeping with the generic quality of the intervention, specifically designed to be adaptable across both typical and atypical development. This was an important practical and ethical issue with this form of preventative work in which families were not selected for the presence of current atypicalities in their child.

Given the small numbers in this case series, any inferences from the results regarding an intervention effect must clearly remain preliminary. However a signal of a potential effect does come two aspects of parental interactive behaviour in the dyad (sensitive responding and nondirectiveness). These represent the theoretical proximal intended outcomes of the intervention and furthermore occur in specific areas in which our separate empirical research has suggested are disrupted in the high-risk group compared to normative controls (Wan et al. 2012a, b). This specificity supports the theory behind the intervention model and its potential utility in work with this high-risk group. Infant liveliness - also found to be reduced in HR compared to LR groups at 8 months in our previous work (Wan et al. 2012a) — showed overall mean improvement across the groups but particularly so in the intervention series.
We consider it also valuable to have shown a similar pattern and trajectory of interaction changes in both the home setting (i.e. in the context where the intervention took place) and also in the one-step-removed context of parentchild play in the laboratory. This gives prime-facie evidence for both the stability of the changes made and also that they may generalise across context from home to lab.

Impact of the intervention on less proximal measures of infant behaviour and cognition shows no simple trends. Data from a laboratory measure of visual attention shows a trend, consistent with some previous literature, in both control groups for a slight decrease in the disengagement effect with increasing age. The one intervention case classified as atypical on this measure at the first visit also showed a reduced disengagement effect at the second test. However, they still remained as an outlier compared to both control groups even at the second test. Clearly, only a larger scale study can address the issue of whether aspects of infant attention can be changed as a result of a parentmediated intervention in a similar way to that demonstrated for infant-directed intervention with typically-developing infants (Wass et al. 2011).

In summary, this case series has demonstrated the feasibility of mounting this early prodromal intervention and its good acceptability to families. It also supports the face validity of our measurement paradigm and gives initial evidence regarding intervention effect in at least the proximal goal for intervention change in our model (i.e. aspects of parental interactive behaviour). Clearly, any inferences from this initial case series are limited by its sample size and design, but these initial findings support our view that a measurement paradigm integrating domains of measurement over time will be well suited to test how the downstream effects of an intervention may play out over development. Further study will need to generate well matched control groups through a random allocation design, and could usefully include a 'contact control' condition of non-specific parent-infant play to test the specificity of the effects targeted by the intervention. One analytic approach 
to testing the efficacy of prodromal intervention in modifying autism outcomes would be to identify meaningful changes in ASD recurrence rates between groups; another more developmental model would be to use multivariate survival methods to assess the effects of the intervention in hastening skill acquisition/normative development or delaying onset of ASD characteristic symptoms.

Acknowledgments We acknowledge with thanks the generous collaboration from the originators of the original VIPP intervention (Femmie Juffer, Marian Bakermans Kranenberg, Marinus van IJzendoorn) that forms the basis of our intervention model. We are grateful for the enormous contributions BASIS families have made towards this study. The research was supported by awards from Autistica, Waterloo Foundation and Central Manchester Foundation NHS Trust to Jonathan Green; from the UK Economic and Social Research Council to Ming Wai Wan and Jonathan Green; Walport Academic Clinical Fellowship to Samina Holsgrove; and from the UK Medical Research Council (G0701484) and the BASIS funding consortium led by Autistica (www.basisnetwork.org) to M.H. Johnson.

\section{References}

Aldred, C., Green, J., Emsley, R., \& McConachie, M. (2011). Brief report: Mediation of treatment effect in a communication intervention for pre-school children with autism. Journal of Autism and Developmental Disorders, 42(3), 447-454.

Bakermans-Kranenburg, M. J., Van Ijzendoorn, M. H., \& Juffer, F. (2003). Less is more: Meta-analyses of sensitivity and attachment interventions in early childhood. Psychological Bulletin, 129(2), 195-215.

Bedford, R., Elsabbagh, M., Gliga, T., Pickles, A., Senju, A., Charman, T., et al. (2012). Precursors to social and communication difficulties in infants at-risk for autism: Gaze following and attentional engagement. Journal of Autism and Developmental Disorders, 42(10), 2208-2218.

Blazey, K. (2007). Attachment in autism: An investigation into parental sensitivity, mutuality and affect. MPhil dissertation, The University of Manchester, Manchester (UK).

Bryson, S. E., Zwaigenbaum, L., McDermott, C., Rombough, V., \& Brian, J. (2008). The autism observation scale for infants: Scale development and reliability data. Journal of Autism and Developmental Disorders, 38(4), 731-738.

Cardoso-Martins, C., \& Mervis, C. B. (1985). Maternal speech to prelinguistic children with Down syndrome. American Journal of Mental Deficiency, 89(5), 451-458.

Charman, T., \& Baird, G. (2002). Practitioner review: Diagnosis of autism spectrum disorder in 2- and 3-year old children. Journal of Child Psychology and Psychiatry, 43(3), 289-305.

Chawarska, K., Klin, A., Paul, R., Macari, S., \& Volkmar, F. (2009). A prospective study of toddlers with ASD: short term diagnostic and cognitive outcomes. Journal of Child Psychology and Psychiatry, 50(10), 1235-1245.

Chawarska, K., Macari, S., \& Shic, F. (2012). Context modulates attention to social scenes in toddlers with autism. Journal of Child Psychology and Psychiatry, 53(8), 903-913.

Crawley, S. B., \& Spiker, D. (1983). Mother-child interactions involving 2-year-olds with down syndrome - a look at individual differences. Child Development, 54(5), 1312-1323.

Dawson, G. (2008). Early behavioural intervention, brain plasticity, and the prevention of autistic spectrum disorder. Development and Psychopathology, 20, 775-803.
Dunst, C. J. (1985). Communicative competence and deficits: Effects on early social interactions. In E. T. McDonald \& D. L. Gallagher (Eds.), Facilitating social-emotional development in multiply handicapped children (pp. 93-140). Philadelphia: Home of the Merciful Saviour for Crippled Children.

Elsabbagh, M., Holmboe, K., Gliga, T., Mercure, E., Hudry, K., Charman, T., et al. (2011). Social and attention factors during infancy and the later emergence of autism characteristics. Progress in Brain Research, 189, 195-207.

Elsabbagh, M., \& Johnson, M. H. (2007). Infancy and autism: Progress, prospects, and challenge. Progress in Brain Research, 164, 355-383.

Elsabbagh, M., \& Johnson, M. H. (2010). Getting answers from babies about autism. Trends in Cognitive Science, 14, 81-87.

Elsabbagh, M., Fernandes, J., Webb, S. J., Dawson, G., Charman, T., Johnson, M. H., et al. (in press). Disengagement of Visual Attention in Infancy Is Associated with Emerging Autism in Toddlerhood. Biological Psychiatry. doi:10.1016/j.biopsych.2012.11.030.

Elsabbagh, M., Mercure, E., Hudry, K., Chandler, S., Pasco, G., Charman, T., et al. (2012). Infant neural sensitivity to eye gaze predicts characteristics of autism at two years. Current Biology, 33, 338-342.

Elsabbagh, M., Volein, A., Tucker, L., Holmboe, K., Csibra, G., Baron-Cohen, S., et al. (2009). Visual orienting in the early broader autism phenotype: Disengagement and facilitation. Journal of Child Psychology and Psychiatry, 50, 637-642.

Flanagan, J. E., Landa, R., Bhat, A., \& Bauman, M. (2012). Head lag in infants at risk for autism: A preliminary study. The American Journal of Occupational Therapy, 66(5), 577-585.

Goodman, R., Ford, T., Richards, H., Gatward, R., \& Meltzer, H. (2000). The development and well-being assessment: Description and initial validation of an integrated assessment of child and adolescent psychopathology. Journal of Child Psychology and Psychiatry, 41, 645-655.

Green, J., Charman, T., McConachie, H., Aldred, C., Slonims, V., Howlin, H., et al. (2010). Parent-mediated communicationfocused treatment in children with autism (PACT): A randomised controlled trial. The Lancet, 375(9732), 2152-2160.

Green, J., \& Dunn, G. (2008). Using intervention trials in developmental psychiatry to illuminate basic science. British Journal of Psychiatry, 192(5), 323-325.

Harris, S., Kasari, C., \& Sigman, M. D. (1996). Joint attention and language gains in children with Down syndrome. American Journal on Mental Retardation, 100(6), 608-619.

Howe, G. W., Reiss, D., \& Yuh, J. (2002). Can prevention trials test theories of etiology? Development and Psychopathology, 14, 673-694.

Johnson, M. H. (2001). Functional brain development in humans. Nature Reviews Neuroscience, 2, 475-483.

Johnson, M. H. (2011). Interactive specialization: A domain-general framework for human functional brain development? Developmental Cognitive Neuroscience, 1, 7-21.

Johnson, M. H., Posner, M. I., \& Rothbart, M. K. (1991). Components of visual orienting in early infancy: Contingency learning, anticipatory looking, and disengaging. Journal of Cognitive Neuroscience, 3, 335-344.

Juffer, F., Bakerman-Kranenburg, M. J., \& Van Ijzendoorm, M. J. (2008). Promoting positive parenting: An attachment-based intervention. New York: Taylor Francis Group.

Kasari, C., Gulsrud, A. C., Wong, C., Kwon, S., \& Locke, J. (2010). Randomized controlled caregiver mediated joint engagement intervention for toddlers with autism. Journal of Autism and Developmental Disorders, 40(9), 1045-1056.

Legerstee, M., Varghese, J., \& Van Beek, Y. (2002). Effects of maintaining and redirecting infant attention on the production of referential communication in infants with and without Down syndrome. Journal of Child Language, 29(1), 23-48. 
Matias, C. S. F. (2006). Direct observations of parent-child interaction based on attachment theory. $\mathrm{PhD}$ thesis, Institute of Psychiatry, King's College, London (UK).

McCathren, R. B., Yoder, P. J., \& Warren, S. F. (1995). The role of directives in early language intervention. Journal of Early Intervention, 19(2), 91-101.

Mullen, E. M. (1995). Mullen scales of early learning. Minnesota: American Guidance Service.

Murray, L., Fiori-Cowley, A., Hooper, R., \& Cooper, P. (1996). The impact of postnatal depression and associated adversity on early mother-infant interactions and later infant outcome. Child Development, 67(5), 2512-2526.

NICHD Early Child Care Research Network. (2000). The relation of child care to cognitive and language development. Child Development, 71(4), 960-980.

Ozonoff, S., Young, G. S., Carter, A., Messinger, D., Yirmya, N., Zwaigenbaum, L., et al. (2011). Recurrence risk for autism spectrum disorders: A baby siblings research consortium study. Pediatrics, 128(3), 2010-2825.

Pickles, A., Charman, T., Green, J., McConachie, H., Aldred, C. R., \& the PACT Consortium. (2011). Identifying therapeutic mechanisms with intervention studies. International Meeting for Autism Research, May 12th 2011. San Diego (USA).

Rozga, A., Hutman, T., Young, G. S., Rogers, S. J., Ozonoff, S., Dapretto, M., et al. (2011). Behavioral profiles of affected and unaffected siblings of children with ASD: Contribution of measures of mother-infant interaction and nonverbal communication. Journal of Autism and Developmental Disorders, 41, 287-301.

Rutter, M., Bailey, A., \& Lord, C. (2003). Social Communication Questionnaire (SCQ). Los Angeles, LA: Western Psychological Services.

Saint-Georges, C., Mahdhaoui, A., Chetouani, M., Cassel, R. S., Laznik, M.-C., Apicella, F., et al. (2011). Do parents recognize autistic deviant behavior long before diagnosis? Taking into account interaction using computational methods. PLOS ONE, 6, $1-13$.

Siller, M., \& Sigman, M. (2002). The behaviors of parents of children with autism predict the subsequent development of their children's communication. Journal of Autism and Developmental Disorders, 32(2), 77-89.

Siller, M., \& Sigman, M. (2008). Modeling longitudinal change in the language abilities of children with autism: parent behaviours and child characteristics as predictors of change. Developmental Psychology, 44(6), 1691-1704.

Slonims, V., Cox, A., \& McConachie, H. (2006). Analysis of motherinfant interaction in infants with Down syndrome and typically developing infants. American Journal on Mental Retardation, 111(4), 273-289.
Sorce, J. F., \& Emde, R. N. (1982). The meaning of infant emotional expressions: Regularities in caregiving responses in normal and Down's syndrome infants. Journal of Child Psychology and Psychiatry and Allied Disciplines, 23(2), 145-158.

Steiner, A. M., Gengoux, G. W., Klin, A., \& Chawarska, K. (2013). Pivotal response treatment for infants at-risk for autism spectrum disorders: A pilot study. Journal of Autism and Developmental Disorders, 43, 91-102.

Walden, T. A., Blackford, J. U., \& Carpenter, K. L. (1997). Differences in social signals produced by children with developmental delays of differing etiologies. American Journal on Mental Retardation, 102(3), 292-305.

Wallace, K. S., \& Rogers, S. J. (2010). Intervening in infancy: Implications for autism spectrum disorders. Journal of Child Psychology and Psychiatry, 51(12), 1300-1320.

Wan, M. W., Green, J., Elsabbagh, M., Johnson, M., Charman, T., Plummer, F., et al. (2012a). Parent-infant interaction in infant siblings at risk of autism. Research in Developmental Disabilities, 33, 924-932.

Wan, M. W., Green, J., Elsabbagh, M., Johnson, M., Charman, T., Plummer, F., et al. (2012b). Quality of interaction between atrisk infants and caregiver at $12-15$ months is associated with 3-year autism outcome. Journal of Child Psychology and Psychiatry. doi:10.1111/jcpp.12032.

Wass, S. V., Porayska-Pomsta, K., \& Johnson, M. H. (2011). Training attentional control in infancy. Current Biology, 21(18), $1543-1547$.

Yirmiya, N., \& Charman, T. (2010). The prodrome of autism: early behavioural and biological signs, regression, peri- and post-natal development and genetics. Journal of Child Psychology and Psychiatry, 51(4), 432-458.

Yirmiya, N., Gamliel, I., Pilowsky, T., Feldman, R., Baron-Cohen, S., \& Sigman, M. (2006). The development of siblings of children with ASD at 4 and 14 months: social engagement, communication, and cognition. Journal of Child Psychology and Psychiatry, 47, 511-523.

Yoder, P. J., \& Warren, S. F. (2004). Early predictors of language in children with and without Down syndrome. American Journal on Mental Retardation, 109(4), 285-300.

Zwaigenbaum, L., Bryson, S. E., Smith, I. M., Szatmari, P., Brian, J., Roberts, W., et al. (2011). A comparison of behavioral markers of ASD in a high-risk infant cohort based on cognitive level at 3 years. International Meeting for Autism Research, May 14th 2011. San Diego, USA.

Zweigenbaum, L., Bryson, S., Rogers, T., Roberts, W., Brian, J., \& Szatmari, P. (2005). Behavioral manifestations of autism in the first year of life. International Journal of Developmental Neuroscience, 23(2-3), 143-152. 